\title{
A System-Level Approach to Overweight and Obesity in the Veterans Health Administration
}

\author{
Susan D. Raffa, PhD' ${ }^{7}$, Matthew L. Maciejewski, PhD ${ }^{2,3}$, Lindsey E. Zimmerman, PhD', \\ Laura J. Damschroder, MS, MPH', Paul A. Estabrooks, PhD ${ }^{6}$, Ronald T. Ackermann, MD, MPH', \\ Adam G. Tsai, MD, MSCE ${ }^{8,9}$, Trina Histon, $P h D^{8}$, and Michael G. Goldstein, $M D^{7}$
}

\begin{abstract}
${ }^{7}$ National Center for Health Promotion and Disease Prevention, Veterans Health Administration, Durham, NC, USA; ${ }^{2}$ Center for Health Services Research in Primary Care, Department of Veterans Affairs Medical Center, Durham, NC, USA; ${ }^{3}$ Division of General Internal Medicine, Department of Medicine, Duke University, Durham, NC, USA; ${ }^{4}$ National Center for PTSD, VA Palo Alto Health Care System, Menlo Park, CA, USA; ${ }^{5}$ VA Ann Arbor Center for Clinical Management Research, Ann Arbor, MI, USA; ${ }^{6}$ College of Public Health, University of Nebraska Medical Center, Omaha, NE, USA; ${ }^{7}$ Northwestern University Feinberg School of Medicine, Chicago, IL, USA; ${ }^{8}$ Kaiser Permanente Care Management Institute, Oakland, CA, USA;

'University of Colorado School of Medicine, Aurora, CO, USA.
\end{abstract}

Healthcare systems are challenged by steady increases in the number of patients who are overweight and obese. Large-scale, evidence-based behavioral approaches for addressing overweight and obesity have been successfully implemented in systems such as the Veterans Health Administration (VHA). These population-based interventions target reduction in risk for obesity-associated conditions through lifestyle change and weight loss, and are associated with modest weight loss. Despite the fact that VHA has increased the overall reach of these behavioral interventions, the number of high-risk overweight and obese patients continues to rise. Recommendations for weight loss medications and bariatric surgery are included in clinical practice guidelines for the management of overweight and obesity, but these interventions are underutilized. During a recent state of the art conference on weight management held by VHA, subject matter experts identified challenges and gaps, as well as potential solutions and overarching policy recommendations, for implementing an integrated system-wide approach for improving population-based weight management.

KEY WORDS: veterans; obesity; weight management; population health. J Gen Intern Med 32(Suppl 1):S79-S82

DOI: $10.1007 / \mathrm{s} 11606-016-3948-\mathrm{Z}$

(c) Society of General Internal Medicine 2016

\section{INTRODUCTION}

Consistent with general trends in the US, ${ }^{1}$ the number of overweight and obese Veterans has steadily increased. ${ }^{2}$ Overweight $^{2}$ and obesity are associated with increased risk of chronic conditions, reduced quality of life, and premature mortality. ${ }^{3}$ In 2006, and updated in 2014, the Department of Veterans Affairs (VA) and Department of Defense (DoD) published an evidencebased Clinical Practice Guideline for Screening and Management of Overweight and Obesity (CPG). ${ }^{2}$ Also in 2006, the Veterans Health Administration (VHA) operationalized several CPG recommendations with implementation of the MOVE! $!^{\circledR}$ Weight Management Program for Veterans (MOVE!), an

Published online March 7, 2017 evidence-based, population-focused approach. ${ }^{4}$ Guided by national policy, MOVE! is a comprehensive lifestyle intervention (CLI) that includes caloric restriction, promotion of physical activity, and behavioral strategies targeting eating and physical activity. Facility-based MOVE! Coordinators and Physician Champions lead program activities. Successful implementation of MOVE! has resulted in near-universal referral of eligible Veterans and clinically significant weight loss for participants. ${ }^{5}$ Nonetheless, rates of overweight and obesity among Veterans treated in VHA have continued to rise, as they have in the general US population. Moreover, VHA has not adopted a system-wide integrated approach linking behavioral weight management with pharmacotherapy and bariatric surgery, as recommended in the CPG.

In 2016, VHA convened a state of the art conference (SOTA) on weight management to evaluate evidence supporting three primary interventions for weight loss (i.e., behavioral, pharmacologic, surgical), identify related research and implementation gaps, and develop priorities for action. Experts in these interventions participated in three workgroups to accomplish SOTA objectives; their respective findings are summarized elsewhere in this supplement. Conference organizers initially planned a fourth workgroup to evaluate evidence for an integrated, coordinated approach to weight management that would support full CPG implementation at the health system level, but were unable to locate evidence for this approach. Instead, all SOTA attendees, including implementation scientists, participated in a concluding session to identify challenges and gaps, as well as potential solutions and policy recommendations for implementing an integrated system-wide approach to weight management in VHA. This paper summarizes workgroup findings regarding challenges and potential solutions in four overlapping areas: implementing an evidence-based multicomponent approach to weight management, providing access to overweight and obesity care across VHA, promoting care coordination and patient engagement strategies, and 
stratifying treatment by level of patient risk. It concludes with workgroup recommendations for establishing an integrated approach to overweight/obesity at a health-system level.

\section{IMPLEMENTING AN EVIDENCE-BASED MULTICOM- PONENT APPROACH TO WEIGHT MANAGEMENT}

Based on systematic reviews of the clinical and epidemiological literature, CPG recommendations included the following: yearly screening and documentation of overweight/obesity; assessment; offering CLI to Veterans who are obese or overweight with an obesity-associated condition; and pharmacotherapy and bariatric surgery as adjuncts to CLI. The CPG also endorsed shared decision-making between providers and patients to support patient engagement based on patient values and preferences. Its recommended algorithm included repeated assessment of treatment adjustments and outcomes to ensure clinically meaningful weight change. Although the CPG emphasized the role of primary care teams, particularly with regard to screening and assessment, the algorithm did not specify how healthcare systems might be organized, or redesigned, to operationalize recommendations and achieve efficient, coordinated management of overweight/obesity.

The workgroup noted gaps in VHA's implementation of key CPG recommendations. First, there is differential uptake of weight loss interventions across VA healthcare facilities. Pharmacotherapy and bariatric surgery are provided to a minority of eligible Veterans, in part because bariatric surgery and pharmacotherapy resources are not closely linked to MOVE!. Moreover, there is variability in referral and use of behavioral (i.e., MOVE!), pharmacologic, and surgical options for addressing obesity. Despite preliminary research on successful implementation features of MOVE! programs, ${ }^{6}$ the underlying mechanisms of system-wide variation in uptake of treatment options and patient outcomes remain unclear. Second, there are significant gaps in understanding decisionmaking related to engaging patients in specific weight management interventions, including who makes decisions and how and when adjustments or transitions to other care modalities are made.

To address gaps in CPG implementation, SOTA participants recommended that VHA develop a national, systemlevel framework for coordinating and integrating delivery of all recommended therapeutic services across clinical settings. This approach must include increased coordination at local/ regional levels to promote efficient use of local resources across clinical service lines.

\section{ACCESS TO OVERWEIGHT AND OBESITY CARE ACROSS VHA}

Given the increasing rates of overweight/obesity and clear evidence of an impact on chronic conditions, there is increased need for effective interventions. Implementation of large-scale programs presents the challenge of ensuring availability of all effective treatment options across facilities for all eligible patients. For example, CLI requires many clinical encounters over time, which is demanding for Veterans and staff. Because of resource limitations, MOVE! may not be available at times and locations or in formats (e.g., individual face-to-face, telephone) that Veterans prefer or require. There is also regional variability in subpopulations of Veterans receiving care in VHA, and the sources of this variability are not fully understood. Facilities vary in size, resource allocation, and care complexity based on demographic, socioeconomic, and general health factors among Veterans in a particular region. In some regions, especially where high percentages of Veterans live in rural areas, and distance to facilities is a barrier, it is not feasible to offer every effective treatment option, thereby reducing the system's potential to meet individual needs. To address these access challenges, VHA must maximize efficient use of existing resources while also identifying and deploying strategies to deliver effective interventions that are scalable and sustainable (e.g., telephonic or telehealth modalities of CLI). ${ }^{7}$

\section{CARE COORDINATION AND PATIENT ENGAGEMENT STRATEGIES}

Care coordination for overweight/obesity requires the functional integration of policies, people, and technologies to ensure that the most effective resources and services are offered to Veterans most likely to benefit. Limited information is available regarding effective patient engagement strategies following assessment and documentation of overweight/obesity. It is unclear what proportion of patients who could benefit from CLI, pharmacologic, or surgical approaches to reduce weight actually engage in discussions of these options with providers in ways that promote patient engagement (e.g., patient-centered counseling, motivational interviewing). Effective, coordinated patient engagement and retention strategies across services, even when services vary in geographic distance or patient contact frequency or type, could reduce obesity-related risk for VHA patients.

Ideally, in an integrated system like VHA, clinicians are aware of which patients are succeeding and which need intensification or change in weight management treatment. Care coordination plans clarify who is responsible for tracking patients and outcomes and initiating treatment changes. In VHA, these responsibilities are not formally assigned to specific providers or services. Consequently, service-line initiatives may lack coordination (e.g., primary care providers screen, provide brief counseling, and refer to MOVE!, whereas bariatric surgery programs are managed by surgical services, and pharmacotherapies may be provided by clinical pharmacists). Coordination across initiatives would optimize each patient's potential to benefit from the range of available services based on individual needs and preferences. 
Primary care teams are considered an appropriate point of care for coordinating outpatient weight management services, but primary care in VHA is subject to numerous evolving mandates, including demands for increased access. Given the prevalence of overweight/obesity in VHA patients, it is unrealistic for such teams to coordinate these services for all eligible patients. Also, the challenge of engaging patients in shared decision-making to help them choose among, and participate in, the full range of weight management interventions, and the iterative nature of assessing outcomes and adjusting treatment plans for individual patients suggest that it will be difficult for primary care teams alone to address the complex needs of overweight/obese Veterans. Though MOVE! Coordinators and Physician Champions have the potential to serve a coordinating function, support for this function is currently limited.

\section{TREATMENT STRATIFICATION BY LEVEL OF PATIENT RISK}

One goal of a population health approach is to engage all Veterans who may benefit from participation in effective interventions to achieve clinically relevant weight loss and reduce risk for obesity-associated conditions. For the population of Veterans who are overweight with an obesity-associated condition or who are obese, MOVE! is effective for achieving clinically significant weight loss in those who participate. ${ }^{6}$ Deliverable in a number of formats, MOVE! enables greater reach to the broad population of overweight/obese Veterans, which should contribute to a population health impact. From this perspective, the availability of MOVE! is critical for the majority of patients.

Population segmentation and thoughtful parsing of services to subgroups based on risk or need is an important aspect of effective population health management. Identifying the majority of patients who could benefit from lower-intensity interventions and the higher-risk patients who could benefit from more intensive treatments is a core strategy. For example, a substantial subset of patients with higher body mass index (BMI) scores and higher short-term risk of developing morbidity from obesity-associated conditions could benefit from adjunctive interventions (i.e., weight loss medications, bariatric surgery) to achieve clinically significant weight loss. Among patients with diabetes and BMI above 35, for instance, bariatric surgery can achieve remission of diabetes ${ }^{8}$ and improve other cardiovascular risk factors. Bariatric surgery also induces significant long-term weight loss 9 and reduces longterm mortality among Veterans. ${ }^{10}$ Thus, targeting higher-risk subpopulations may be more likely to produce measurable reductions in morbidity in the near term.

A population health approach that balances high-reach strategies for modest benefits among many, with identification and focus on subsets of higher-risk patients in need of greater intervention, requires weight management services that are integrated and coordinated at the health system level. This includes providing resources to track Veteran participation in interventions in real time, identifying those who require additional intervention, and supporting communication across providers of all interventions (i.e., across surgical teams and teams providing MOVE! and pharmacotherapies).

\section{RECOMMENDATIONS AND CONCLUSIONS}

A CPG for screening and management of overweight/obesity is implemented to varying degrees across VA healthcare facilities due to the absence of an integrated, coordinated health systemlevel framework. Such a framework is needed to inform how different parts of the system may best work together to address population health, while also coordinating the continuum of weight management care over a patient's lifetime. As a national health system, VHA has an opportunity to integrate behavioral, pharmacologic, and surgical interventions to improve populationbased weight management. This could be facilitated by leveraging pre-existing implementation resources (e.g., electronic medical record, MOVE! CLI programming) to support care coordination and to track population-, program-, and patient-level outcomes.

We recommend the following system-level strategies to facilitate development and implementation of an integrated framework for management of overweight/obesity in VHA:

- Establish an organization-wide priority of addressing overweight/obesity as a population health goal. This may require a cultural shift or a narrowing of focus as all levels of VHA prioritize this goal.

- Assess impact with a population-based indicator (e.g., proportion of patients achieving 5\% weight loss).

- Establish a clear governance structure to coordinate weight management care at the health system level. Currently, three separate system-level policies guide implementation of behavioral, pharmacologic, and surgical weight management interventions. A governance structure that establishes an integrated national policy would ensure that infrastructure and resources are available to coordinate and integrate weight management care across service lines. Infrastructure and coordination/communication channels that bridge other clinical services (e.g., nursing, nutrition) are also needed. For example, additional staff could be supported in implementing patient engagement strategies in collaboration with primary care providers and care coordination nurses who care for patients with obesity-associated chronic conditions (e.g., diabetes, hypertension).

- Implement large-scale measurement strategies for identifying at-risk patients and matching them with the most appropriate services, tracking patients over time, and evaluating treatment fidelity, quality, and associated outcomes. This will also allow for identification of facilities that successfully support high proportions of patients achieving clinically meaningful 
weight loss, as well those with lower success rates that could be offered quality improvement strategies to enhance impact.

In conclusion, a system-level framework for integrated, coordinated weight management is needed in VHA to fully implement the CPG and improve Veteran health outcomes. If the VHA is successful in adopting a fully integrated, coordinated population-based approach, it not only has the potential to improve Veteran health and well-being; it may offer other healthcare systems a model that "bends the curve" of the obesity epidemic.

\section{Acknowledgements:}

Contributors: None

Corresponding Author: Susan D. Raffa, PhD; National Center for Health Promotion and Disease PreventionVeterans Health Administration, Durham, NC, USA (e-mail: susan.raffa@va.gov).

Compliance with Ethical Standards:

Funders: The state of the art conference was supported by the Department of Veterans Affairs, Veterans Health Administration, Office of Research and Development, Health Services Research and Development (HSR\&D). Dr. Maciejewski received support from VA HSR\&D for a Research Career Scientist Award (RCS 10-391). Ms. Damschroder received support from VA HSR\&D Quality Enhancement Research Initiative (VA QUE 15-286).

\section{Prior Presentations: None}

Conflict of Interest: Adam Tsai has received honoraria from Vindico Medical Education and Med-IQ. All other authors declare that they have no conflict of interest.
Disclaimer: The views expressed in this article are those of the authors and do not necessarily reflect the position or policy of the Veterans Health Administration, Department of Veterans Affairs, or the US Government.

\section{REFERENCES}

1. Flegal KM, Kruszon-Moran D, Carroll MD, Fryar CD, Ogden CL. Trends in obesity among adults in the United States, 2005 to 2014. JAMA. 2016;315:2284-2291.

2. VA/DoD. 2014 Clinical Practice Guideline for the Screening and Management of Overweight and Obesity. Available at: http://www. healthquality.va.gov/guidelines/cd/obesity/. Accessed July 18, 2016.

3. Jensen MD, Ryan DH, Apovian CM, et al. AHA/ACC/TOS guideline for the management of overweight and obesity in adults: a report of the American College of Cardiology/American Heart Association Task Force on Practice Guidelines and The Obesity Society. J Am Coll Cardiol. 2014;63(25 Pt B):2985-3023.

4. Kinsinger LS, Jones KR, Kahwati L, et al. Design and dissemination of the MOVE! weight-management program for veterans. Prev Chronic Dis. 2009;6:A98.

5. Littman AJ, Damschroder LJ, Verchinina L, et al. National evaluation of obesity screening and treatment among veterans with and without mental health disorders. Gen Hosp Psychiatry. 2015;37:7-13.

6. Kahwati LC, Lance TX, Jones KR, Kinsinger LS. RE-AIM evaluation of the Veterans Health Administration's MOVE! Weight Management Program. Transl Behav Med. 2011;1:551-60.

7. Pronk NP. Designing and Evaluating Health Promotion Programs. Dis Manage Health Outcomes. 2003;11:149-157.

8. Arterburn DE, Courcoulas AP. Bariatric surgery for obesity and metabolic conditions in adults. BMJ. 2014;349:g3961.

9. Maciejewski ML, Arterburn DE, Van Scoyoc L, et al. Bariatric surgery and long-term durability of weight loss. JAMA Surg [Internet]. 2016 Aug 31[cited 2016 Nov 2]: [10 p.]. Available from: http://jamanetwork. com/journals/jamasurgery/fullarticle/2546331

10. Arterburn DE, Olsen MK, Smith VA, et al. Association between bariatric surgery and long-term survival. JAMA. 2015;313:62-70. 